\title{
Even-odd transition in the Shubnikov-de Haas oscillations in a two-dimensional electron gas subjected to periodic magnetic and electric modulations
}

\author{
Jirong Shi and F. M. Peeters* \\ Departement Natuurkunde, Universiteit Antwerpen (UIA), Universiteitsplein 1, B-2610 Antwerpen, Belgium \\ K. W. Edmonds and B. L. Gallagher \\ School of Physics and Astronomy, University of Nottingham, Nottingham NG7 2RD, United Kingdom
}

(Received 1 October 2001; published 24 July 2002)

\begin{abstract}
We investigate low-temperature magnetotransport of high-mobility two-dimensional electron gases subjected to one-dimensional periodic magnetic and electric modulations. Our previous quantum perturbation theory is extended to lower temperatures and the energy broadening due to impurity scattering is incorporated. Numerical calculations are made for situations where several Landau bands overlap. We find that the Shubnikov-de Haas ( $\mathrm{SdH}$ ) oscillations are dominated by collisional resistance. The amplitudes of the $\mathrm{SdH}$ oscillations are strongly modulated and the positions of the $\mathrm{SdH}$ minima switch between even and odd Landau-level filling factors, in the resistance both parallel and perpendicular to the one-dimensional modulation. This is a consequence of the internal structure (i.e., smeared out van Hove singularities) of overlapping Landau bands. Our theoretical results are in good agreement with recent experiments.
\end{abstract}

DOI: 10.1103/PhysRevB.66.035328

PACS number(s): 72.20.My, 73.23.-b, 73.40.-c

\section{INTRODUCTION}

Two-dimensional electron gases (2DEG's) in highmobility semiconductor heterostructures subjected to a perpendicular magnetic field show interesting transport properties at low temperatures. ${ }^{1}$ The magnetoresistance of a 2 DEG in a uniform magnetic field exhibits Shubnikov-de Haas $(\mathrm{SdH})$ oscillations, due to the changing occupation of the highly degenerate Landau levels in the vicinity of the Fermi energy. The presence of an additional spatially and periodically modulated field (electric or magnetic) results in Weiss oscillations (also known as commensurability oscillations). This phenomenon was first observed a decade ago in GaAs/ AlGaAs heterostructures with submicron-scale onedimensional (1D) periodic electric modulations. ${ }^{2-4}$ The Weiss oscillations can be well understood within a quantummechanical description ${ }^{3-7}$ in which the modulation broadens the Landau levels into bands by lifting their degeneracy with respect to the center coordinate of the wave function. The bandwidth of these Landau bands oscillates as a function of the Landau-level index and magnetic field, due to the commensurability between the modulation period $a$ and the cyclotron diameter at the Fermi energy $2 R_{c}=2\left(2 \pi n_{e}\right)^{1 / 2} l^{2}$, with $l=(\hbar / e B)^{1 / 2}$ the magnetic length and $n_{e}$ the electron density. The group velocity of these bands is perpendicular to the modulation direction, which leads to a diffusion conductivity (also called band conductivity), contributing to the Weiss oscillations in the magnetoresistance parallel to the modulation direction $\left(R_{x x}\right)$. The modulated density of states (DOS) gives rise to oscillations in the collisional conductivity (also called hopping conductivity) and explains the weak Weiss oscillations observed in the resistance perpendicular to the modulation $\left(R_{y y}\right)$. The Weiss oscillations in $R_{x x}$ can also be explained as being a semiclassical effect: ${ }^{8-11}$ they are due to the guiding center drift of the cyclotron orbits in the modulated electric field. However, these semiclassical theories ${ }^{8-11}$ cannot account for the Weiss oscillations in $R_{y y}$ and the SdH oscillations in both $R_{x x}$ and $R_{y y}$, which have a pure quantum-mechanical origin.

The Weiss oscillations with a periodic 1D magnetic modulation were predicted ${ }^{12}$ and investigated theoretically in several works. ${ }^{13-16}$ Successful experiments were realized only in recent years. ${ }^{17-21}$ In these experiments the modulation fields are produced by ferromagnetic or superconducting strips placed on the surface of GaAs/AlGaAs heterostructures. Usually these metallic strips also induce an electric modulation in the 2DEG due to the differential thermal contraction of the deposited metal and the underlying semiconductor at low temperatures. ${ }^{22-24}$ This stress-induced electric modulation is important in understanding the experimental results with periodic magnetic modulations. Very recently Edmonds et al. ${ }^{25}$ made a detailed investigation of the $\mathrm{SdH}$ oscillation of 2DEG's with 1D periodic magnetic modulations. Several interesting phenomena were observed: (1) the $\mathrm{SdH}$ oscillation minima in $R_{x x}$ occur at even Landau-level filling factors $\left(n_{e} h / e B\right)$ near the Weiss oscillation minima and at odd filling factors around Weiss oscillation peaks, (2) the positions of the $\mathrm{SdH}$ minima in $R_{y y}$ switch between even and odd filling factors, and (3) the amplitude of the $\mathrm{SdH}$ oscillations in $R_{x x}$ is found to be a maximum at the minima of the Weiss oscillations. Note that for a unmodulated 2DEG the $\mathrm{SdH}$ oscillations always exhibit a minimum at even filling factors. A related even-odd transition was also observed at magnetic fields beyond the range of the Weiss oscillations with a periodic electric modulation. ${ }^{26}$

In this paper we present a theoretical investigation of the magnetoresisitivity of a $2 \mathrm{DEG}$ subjected to $1 \mathrm{D}$ periodic magnetic and electric fields. We extend our previous quantum perturbation theory ${ }^{5,14}$ to the regime where Landau bands overlap. The broadening effect due to, e.g., scattering with impurities is also included and is important in experiments at low temperatures. The observed switching of the $\mathrm{SdH}$ oscillations between even and odd filling factors is re- 
produced, and shown to be a direct consequence of the internal structure of the Landau bands. It is shown that the strong $\mathrm{SdH}$ oscillations around the Weiss oscillation minima are due to the collisional conductivity contribution. Our results are in good agreement with the experimental observations.

The paper is organized as follows. In Sec. II the electron states and DOS in 1D magnetic and electric fields are presented. We calculate conductivities and resistivities in Sec. III. Comparisons with experimental results are presented in Sec. IV. Concluding remarks are given in Sec. V. In the Appendix we present the calculation of the fringe magnetic field resulting from periodic ferromagnetic strips.

\section{ELECTRON STATES}

We consider a 2DEG in the $(x, y)$ plane subjected to a perpendicular periodic magnetic field $\vec{B}=\left[B_{0}\right.$ $\left.+B_{1} \cos (K x)\right] \vec{e}_{z}$, where $K=2 \pi / a$ and $a$ is the period. In the experiments considered, this $1 \mathrm{D}$ modulation is produced by an array of ferromagnetic strips which are placed a distance $z$ above the 2DEG. The corresponding magnetic field profiles are calculated in the Appendix and can be approximated by a cosine function. In most experiments this magnetic field modulation is accompanied by a 1D periodic electric field with corresponding potential $V(x)=V_{0} \cos (K x)$. This electric modulation is unavoidable due to the mechanical stress of the metallic strips on the underlying semiconductor. In the experiments discussed in Sec. IV, the magnetic and electric modulations are in phase. In the Landau gauge the vector potential is $\vec{A}=\left(0, B_{0} x+\left(B_{1} / K\right) \sin (K x), 0\right)$. In the effectivemass approximation the one-electron Hamiltonian can be written as

$$
H=\frac{1}{2 m^{*}}(\vec{p}+e \vec{A})^{2}+V(x)=H_{0}+H_{1},
$$

where

$$
\begin{gathered}
H_{0}=\left[p_{x}^{2}+\left(p_{y}+e B_{0} x\right)^{2}\right] / 2 m^{*}, \\
H_{1}=\left(\omega_{1} / K\right)\left(p_{y}+e B_{0} x\right) \sin (K x) \\
+\left(m^{*} \omega_{1}^{2} / 2 K^{2}\right) \sin ^{2}(K x)+V_{0} \cos (K x),
\end{gathered}
$$

and $\omega_{1}=e B_{1} / m^{*}$. Here $m^{*}$ is the effective mass of the electrons, and we set $m^{*}=0.067 m_{e}$ as for electrons in GaAs/ AlGaAs heterostructures. $H_{0}$ is the Hamiltonian of a free electron in a uniform magnetic field $B_{0}$. The corresponding eigenstates are $\left|n k_{y}\right\rangle=L_{y}^{-1 / 2} \exp \left(i k_{y} y\right) \phi_{n}\left(x-x_{0}\right)$, where $x_{0}=$ $-k_{y} l^{2}$ is the center coordinate of the cyclotron orbit, $l$ $=\left(\hbar / e B_{0}\right)^{1 / 2}$, and $L_{y}$ is the width of the sample in the $y$ direction. $\phi_{n}(x)$ are the harmonic-oscillator wave functions and the corresponding eigenvalues are $E_{n}^{0}=(n+1 / 2) \hbar \omega_{c}$ and are degenerate with respect to $k_{y}$. In the weakmodulation case $\left|B_{1}\right| \ll\left|B_{0}\right|$ and $V_{0} \ll E_{F}$, one can treat $H_{1}$ as a perturbation and omit the second term in Eq. (2b) which is proportional to $B_{1}^{2}$. The energy with first-order corrections reads

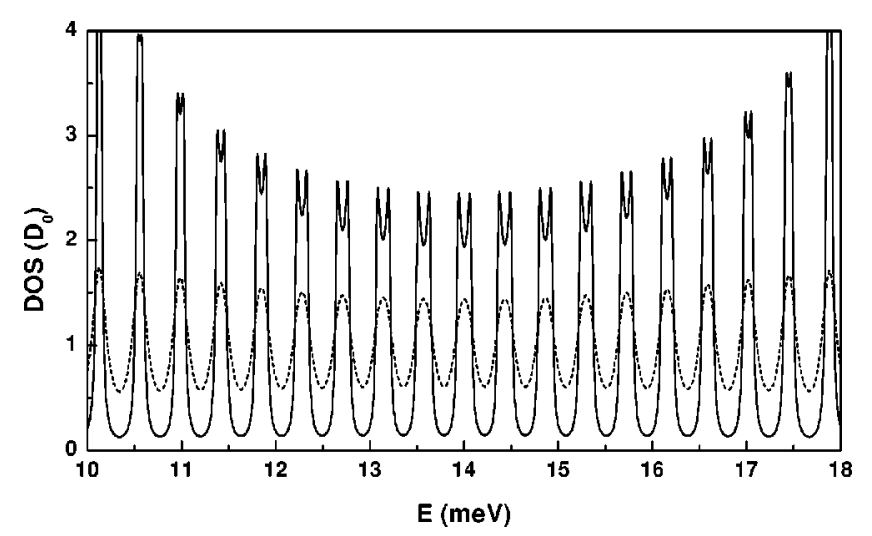

FIG. 1. The DOS (in unit of $D_{0}$ ) of a 2DEG in a 1D modulated magnetic field $B=B_{0}+B_{1} \cos (2 \pi x / a)$ with $B_{0}=0.25 \mathrm{~T}, B_{1}$ $=0.08 B_{0}$, and $a=300 \mathrm{~nm}$. Here $D_{0}=m * / \pi \hbar^{2}$ is the DOS in zero magnetic field. The energy broadening due to impurity scattering $\Gamma$ is $0.2 \mathrm{~K}$ for the solid curve and $1 \mathrm{~K}$ for the dashed curve.

$$
E_{n k_{y}}=(n+1 / 2) \hbar \omega_{0}+\left[\hbar \omega_{1} G_{n}(u)+V_{0} F_{n}(u)\right] \cos \left(K x_{0}\right)
$$

where $\omega_{0}=e B_{0} / m^{*}$ is the cyclotron frequency, $u=K^{2} l^{2} / 2$, $G_{n}(u)=e^{-u / 2}\left[L_{n}^{1}(u)+L_{n-1}^{1}(u)\right] / 2, \quad F_{n}(u)=e^{-u / 2} L_{n}^{0}(u)$, and $L_{n}^{\alpha}(u)$ is the Laguerre polynomial. Since $x_{0}=-k_{y} l^{2}$, the $k_{y}$ degeneracy of the energy levels is lifted and the energy levels broaden into Landau bands. The bandwidth $W_{n}\left(B_{0}\right)$ $=2\left|\hbar \omega_{1} G_{n}(u)+V_{0} F_{n}(u)\right|$ oscillates with magnetic field $B_{0}$ and Landau index $n$. The flatband condition is given by $W_{n}$ $=0$.

In the absence of modulation the DOS consists of a series of $\delta$ functions, $D(E)=\left(1 / 2 \pi l^{2}\right) \Sigma_{n} \delta\left(E-E_{n}^{0}\right)$, with $1 / 2 \pi l^{2}$ states in each Landau level per unit surface. With 1D electric or magnetic modulation the $\delta$ functions in the DOS are broadened into bands with van Hove singularities at the edges. ${ }^{4,6,7,16}$ In practical systems there is always some broadening present due to scattering centers. For simplicity we assume a Lorentzian broadening of the energy with zero shift and constant width $\Gamma, \delta(E) \rightarrow P(E)=(\Gamma / \pi) /\left(E^{2}+\Gamma^{2}\right)$. Introducing the dimensionless variable $t=K x_{0}$, the DOS takes the form

$$
D(E)=D_{0} \hbar \omega_{c} \sum_{n=0}^{\infty} \frac{1}{\pi} \int_{0}^{\pi} d t P\left(E-E_{n t}\right),
$$

where $D_{0}=m^{*} / \pi \hbar^{2}$ is the DOS of a 2DEG at $B=0$. A spin degeneracy factor of 2 is included in Eq. (4).

The energy broadening effect on the DOS is shown in Fig. 1. We calculate DOS with Eq. (4) at magnetic field $B_{0}$ $=0.25 \mathrm{~T}$ with a modulation strength $B_{1}=0.08 B_{0}$. The period of the modulation is $a=300 \mathrm{~nm}$. The energy broadening $\Gamma$ is $0.2 \mathrm{~K}$ for the solid curve and $1 \mathrm{~K}$ for the dashed curve. Periodic changes in the bandwidth (only one period is shown in Fig. 1) and van Hove singularities at the band edges can be clearly seen in the solid curve for which $\Gamma$ is small. The amplitude of the DOS peaks also changes periodically since each Landau band contains the same number of states $\left(1 / 2 \pi l^{2}\right)$. However, as $\Gamma \geqslant W_{n}$ the van Hove singu- 


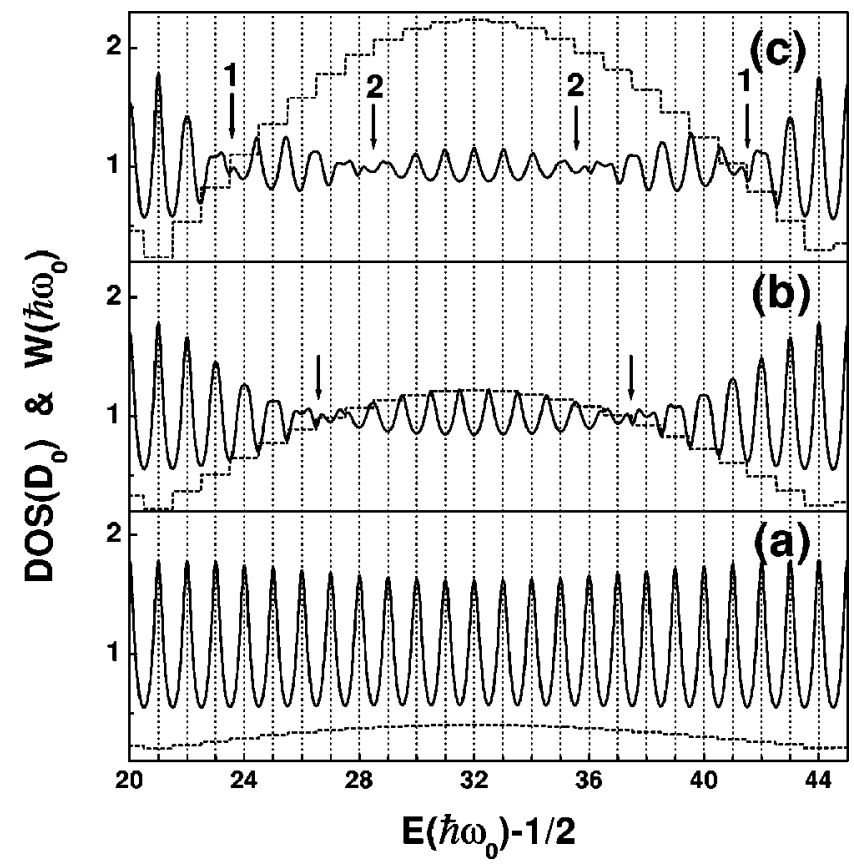

FIG. 2. The DOS (solid curves) and the total bandwidth $W$ $=W_{n}+\Gamma$ (dashed curves) with magnetic modulation strength (a) $B_{1}=0.05 B_{0}$, (b) $B_{1}=0.25 B_{0}$, and (c) $B_{1}=0.5 B_{0}$. The applied magnetic field is $B_{0}=0.25 \mathrm{~T}$, period of the modulation $a=300 \mathrm{~nm}$, and the scattering broadening $\Gamma=1 \mathrm{~K}$. The positions of the Landau levels are indicated by the grid lines.

larities disappear and the DOS exhibits a peak at each band center, as shown by the dashed curve. The amplitude of the DOS oscillation with a larger $\Gamma$ is reduced. As the modulation strength increases the Landau bandwidth $W_{n}$ increases proportionally.

When $W_{n}$ is larger than the cyclotron energy $E_{c}=\hbar \omega_{0}$, adjacent Landau bands overlap. This interesting situation is shown in Fig. 2 where we plot the DOS (solid curves) and the total bandwidth $W=W_{n}+\Gamma$ (dashed curves) as a function of the dimensionless energy $\epsilon=E / E_{c}-1 / 2$. The vertical grid lines are at integer values of $\epsilon$ and indicate the position of the Landau levels. Figure 2(a) shows the case where the modulation $B_{1}=0.05 B_{0}$ is weak and the bandwidth is smaller than $E_{c}$. The DOS exhibits peaks at the position of the unperturbed Landau levels as in the case of a uniform magnetic field. In Fig. 2(b) the modulation is made stronger, $B_{1}=0.25 B_{0}$. In the regime $27 \leqslant \epsilon \leqslant 37$ the bandwidth $W$ is larger than $E_{c}$ and adjacent Landau bands overlap. This is shown more clearly in Fig. 3(a) where we plot the DOS around $\epsilon=32$ (solid curve) and DOS contributions from individual bands with index $n=31$ (dotted curve), $n=32$ (dashed curve), and $n=33$ (dash-dotted curve) respectively. The superposition of adjacent band edges at half integers of $\epsilon$ results in peaks between the Landau levels. The switching between the occurrence of the DOS peaks at the position of the Landau levels and those between Landau levels takes place at the points where $W=E_{c}$, which is marked by the arrows in Fig. 2(b). The amplitude of the DOS oscillations is suppressed around these switching points. In Fig. 2(c) the modulation $B_{1}=0.5 B_{0}$ is so large that the bandwidth $W$ is

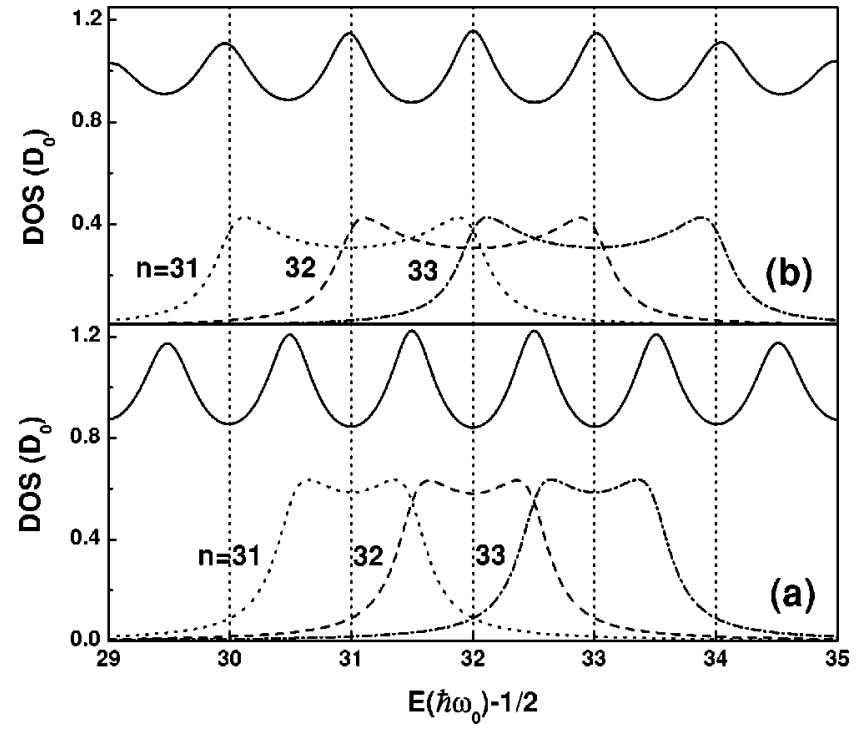

FIG. 3. The DOS (solid curves) and individual contributions from the Landau bands $n=31$ (dotted curves), $n=32$ (dashed curves), and $n=33$ (dash-dotted curves). In (a) the overlapping of two adjacent bands results in DOS peaks between Landau levels. In (b) three neighboring bands overlap. The DOS peaks switch back to Landau-level centers.

larger than $2 E_{c}$ when $29 \leqslant \epsilon \leqslant 35$. In Fig. 3(b) the DOS around $\epsilon=32$ (solid curve) and individual contributions from nearest Landau bands $n=31$ (dotted curve), $n=32$ (dashed curve), and $n=33$ (dash-dotted curve) are plotted. In this region the band edges cross the centers of their adjacent bands and overlap with their next-nearest-neighboring bands. Therefore, three Landau bands overlap at the band centers, and the DOS peaks switch back to integer $\epsilon$. The arrows labeled 1 and 2 in Fig. 2(c) mark the switching points where $W / E_{c}=1$ and 2 , respectively. DOS peaks are present at half integers in the intervals $24 \leqslant \epsilon \leqslant 28$ and $36 \leqslant \epsilon \leqslant 41$ where 1 $<W / E_{c}<2$. Similar behavior can be found when more Landau bands overlap; i.e., when the overlapping band number is odd (even) the DOS has peaks at (between) the center of the Landau levels. The amplitude of the DOS oscillations is diminished as more bands overlap.

The chemical potential $\mu^{*}$ is determined by the electron density $n_{e}=\int_{-\infty}^{\infty} d E f(E) D(E)$, where $f(E)$ is the FermiDirac distribution function. Using Eq. (4) for $D(E)$ we obtain

$$
n_{e} \pi l_{B}^{2}=\sum_{n=0}^{\infty} \frac{1}{\pi} \int_{-\infty}^{\infty} d E \int_{0}^{\pi} d t \frac{1}{e^{\beta\left(E-\mu^{*}\right)}+1} P\left(E-E_{n t}\right)
$$

where $\beta=1 / k_{B} T$ and $T$ is the temperature. Equation (5) is solved numerically to obtain the chemical potential (or the Fermi energy). In Fig. 4(a) we plot the DOS at the Fermi energy $\left(E_{F}\right)$ against the Landau-level filling factor, $\nu$ $=n_{e} h / e B_{0}$. The peak positions in the $D\left(E_{F}\right)$ switch between even and odd filling factors at the points indicated by the arrows. As $\nu<59$ or $\nu>77, D\left(E_{F}\right)$ peaks occur at odd integers of $\nu$; i.e., the DOS reaches its maximum when the Lan- 


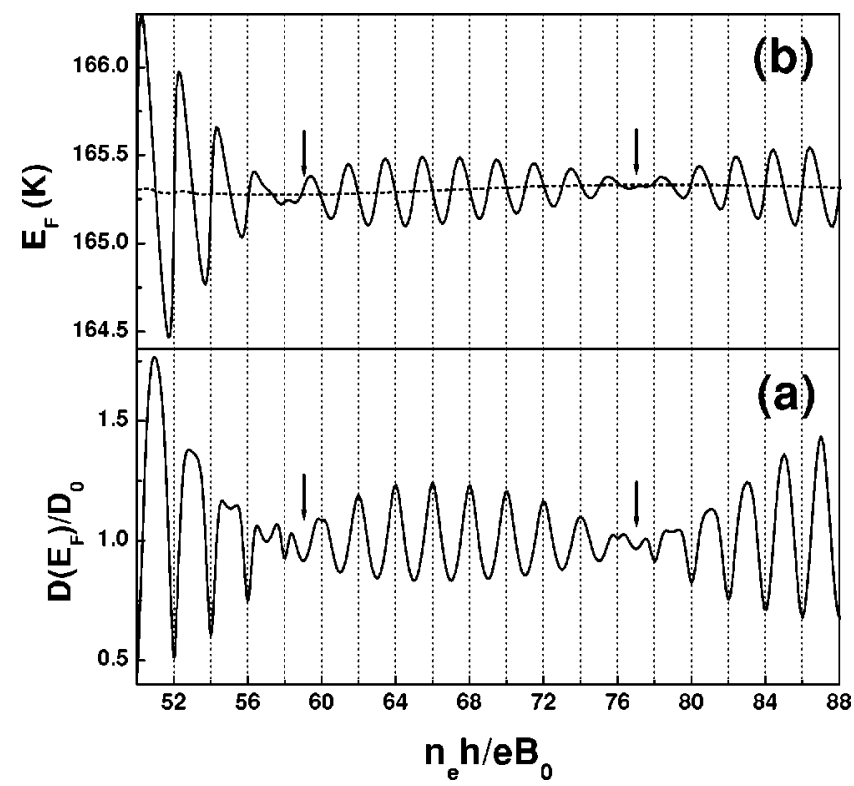

FIG. 4. (a) The DOS at the Fermi energy $E_{F}$ and (b) $E_{F}$ vs the Landau-level filling factor, $\nu=n_{e} h / e B_{0}$, at temperatures $T$ $=0.1 \mathrm{~K}$ (solid curve) and $2 \mathrm{~K}$ (dashed curve). The magnetic modulation strength is $B_{1}=0.12 B_{0}$, energy broadening $\Gamma=1 \mathrm{~K}$, period $a=500 \mathrm{~nm}$, and electron density $n_{e}=4 \times 10^{15} \mathrm{~m}^{-2}$.

dau bands are half filled. Note that a spin degeneracy of 2 is considered in the calculation. As $59<\nu<77$ neighboring bands overlap $\left(1<W / E_{c}<2\right)$ and the DOS has peaks at even integers. In Fig. 4(b), $E_{F}$ as a function of $\nu$ is plotted. At a low temperature $T=0.1 \mathrm{~K}$, as shown by the solid curve, the phase of the $\mathrm{SdH}$ oscillation at the Landau levels switches between 0 and $\pi$ at the same $\nu$ as in $D\left(E_{F}\right)$. At a higher temperature $T=2 \mathrm{~K}$, as shown by the dashed curve in Fig. 4(b), the $\mathrm{SdH}$ oscillations in $E_{F}$ are smeared out by thermal broadening.

\section{CONDUCTIVITIES AND RESISTIVITIES}

The conductivity tensor in the linear response regime was obtained in Ref. 5 from a quantum Boltzmann equation. ${ }^{27}$ In general, $\sigma_{\mu \nu}=\sigma_{\mu \nu}^{d}+\sigma_{\mu \nu}^{n d}$, and $\sigma_{\mu \nu}^{d}=\sigma_{\mu \nu}^{d i f}+\sigma_{\mu \nu}^{c o l}, \mu, \nu=x$, $y, z$. Here $\sigma_{\mu \nu}^{d}\left(\sigma_{\mu \nu}^{n d}\right)$ stems from the diagonal (nondiagonal) part of the current density. $\sigma_{\mu \nu}^{d i f}$ indicates diffusion contributions and $\sigma_{\mu \nu}^{c o l}$ collisional contributions. In this work we include the impurity broadening effect on the conductivity, which is important at low temperatures. For dc conduction, the diffusion (or band) conductivity reads

$$
\sigma_{\mu \nu}^{d i f}=\frac{\beta e^{2}}{A} \sum_{\zeta} \int d E P\left(E-E_{\zeta}\right) f_{E}\left(1-f_{E}\right) \tau(E) v_{\mu}^{\zeta} v_{\nu}^{\zeta},
$$

where $A$ is the area of the sample, $\tau(E)$ is the momentum relaxation time. $v_{\mu}^{\zeta}=\left\langle\zeta\left|v_{\mu}\right| \zeta\right\rangle$ is the group velocity which vanishes for localized states. The collisional (or hopping) conductivity describes transport through localized states due to impurity scattering. It is given by

$$
\begin{aligned}
\sigma_{\mu \mu}^{c o l}= & \frac{\beta e^{2}}{2 A} \sum_{\zeta \zeta^{\prime}} \int d E \int d E^{\prime} P\left(E-E_{\zeta}\right) P\left(E^{\prime}-E_{\zeta^{\prime}}\right) \\
& \times f_{E}\left(1-f_{E^{\prime}}\right) W_{\zeta \zeta^{\prime}}\left(\alpha_{\mu}^{\zeta}-\alpha_{\mu}^{\zeta^{\prime}}\right)^{2},
\end{aligned}
$$

where $W_{\zeta \zeta^{\prime}}$ is the transition rate between $|\zeta\rangle$ and $\left|\zeta^{\prime}\right\rangle$, and $\alpha_{\mu}^{\zeta}=\left\langle\zeta\left|r_{\mu}\right| \zeta\right\rangle$. The nondiagonal conductivity reads

$$
\begin{aligned}
\sigma_{\mu \nu}^{n d}= & \frac{i \hbar e^{2}}{A} \sum_{\zeta \neq \zeta^{\prime}} \int d E \int d E^{\prime} P\left(E-E_{\zeta}\right) P\left(E^{\prime}-E_{\zeta^{\prime}}\right) \\
& \times\left(f_{E}-f_{E^{\prime}}\right) \frac{\left\langle\zeta\left|v_{\mu}\right| \zeta^{\prime}\right\rangle\left\langle\zeta^{\prime}\left|v_{\nu}\right| \zeta\right\rangle}{\left(E-E^{\prime}\right)^{2}} .
\end{aligned}
$$

To evaluate the resistivity tensor $\rho_{\mu \nu}(\mu, \nu=x, y)$ we will use the conductivity tensor $\sigma_{\mu \nu}$ in the standard expression: $\rho_{x x}$ $=\sigma_{y y} / S, \quad \rho_{y y}=\sigma_{x x} / S$, and $\rho_{x y}=-\sigma_{y x} / S$, where $S$ $=\sigma_{x x} \sigma_{y y}-\sigma_{x y} \sigma_{y x}$ and $S \approx \sigma_{x y}^{2}=\left(n_{e} e / B_{0}\right)^{2}$ in the experiments under consideration. The resistance tensor $R_{\mu \nu}$ is the resistivity tensor multiplied by the aspect ratio of the sample.

In the case of weak modulations perturbation theory is effective. We use $|\zeta\rangle=\left|n k_{y}, s\right\rangle=\left|n k_{y}\right\rangle|s\rangle$ as the unperturbed electron state, where $|s\rangle=\left| \pm \frac{1}{2}\right\rangle$ is the wave function associated with the spin. Since we are interested in weak magnetic fields $(B \leqslant 1 \mathrm{~T})$, spin splitting is neglected. The velocity of the electron is calculated from $\vec{v}=\hbar^{-1} \partial E_{n k_{y}} / \partial \vec{k}$. From Eq. (3) we obtain $v_{x}=0$ and

$$
v_{y}=\frac{2 u}{\hbar K}\left[\hbar \omega_{1} G_{n}(u)+V_{0} F_{n}(u)\right] \sin (t) .
$$

For convenience we assume a constant momentum relaxation time $\tau(E)=\tau$ independent of energy, which is a good approximation at weak magnetic fields. Thus the diffusion conductivities $\sigma_{x x}^{\text {dif }}=0$ and

$$
\begin{aligned}
\sigma_{y y}^{d i f}= & \sigma_{0} \int_{-\infty}^{\infty} d E\left(-\frac{\partial f}{\partial E}\right) \sum_{n=0}^{\infty}\left|\hbar \omega_{1} G_{n}(u)+V_{0} F_{n}(u)\right|^{2} \\
& \times \int_{0}^{\pi} d t P\left(E-E_{n t}\right) \sin ^{2} t
\end{aligned}
$$

where $\sigma_{0}=\left(2 e^{2} / h\right)(4 \pi \tau / \hbar)(l / a)^{2}$. In Eq. (10) the modulation-induced Landau-band broadening and the impurity scattering broadening effects are included through integration over $t$ and $E$, respectively. If we omit impurity broadening, $P\left(E-E_{n t}\right) \rightarrow \delta\left(E-E_{n t}\right)$, and using the approximation $(-\partial f / \partial E)_{E_{n t}} \approx(-\partial f / \partial E)_{E_{n}^{0}}$, which is valid when the thermal broadening is larger than the bandwidths, $k_{B} T \gg W_{n}$, we obtain

$$
\begin{aligned}
\sigma_{y y}^{d i f} \approx & \frac{2 e^{2}}{h} \frac{2 \pi^{2} \tau}{\hbar} \frac{l^{2}}{a^{2}} \sum_{n=0}^{\infty}\left|\hbar \omega_{1} G_{n}(u)+V_{0} F_{n}(u)\right|^{2} \\
& \times\left(-\frac{\partial f}{\partial E}\right)_{E=E_{n}^{0}},
\end{aligned}
$$


which is Eq. (22) of Ref. 14 multiplied by 2, a spin degeneracy factor.

We assume that electrons are scattered elastically by randomly distributed impurities. This type of scattering is dominant at low temperatures. The impurity is described by a screened Coulomb potential $U(\vec{r}-\vec{R})=\left(e^{2} / \epsilon|\vec{r}-\vec{R}|\right) \exp$ $\left(-k_{s}|\vec{r}-\vec{R}|\right)$, where $\vec{r}$ and $\vec{R}$ are the position of the electron and impurity, respectively, $k_{s}$ is the inverse screening length, and $\epsilon$ is the dielectric constant. A detailed evaluation of the transition rate $W_{\zeta \zeta^{\prime}}$ can be found in Ref. 7. The energy broadening due to impurity scattering reads $\Gamma=\hbar / \tau_{\zeta}$ $=\hbar \Sigma_{\zeta^{\prime}} \int d E P\left(E-E_{\zeta^{\prime}}\right) W_{\zeta \zeta^{\prime}} \approx\left(N_{I} U_{0}^{2} / \pi l^{2}\right)^{1 / 2}$, with $N_{I}$ the impurity density and $U_{0}=2 \pi e^{2} / \epsilon k_{s}$.

As Landau bands overlap hopping between Landau bands is possible. However, the transition rate $W_{\zeta \zeta^{\prime}}$ between states in different Landau bands is much smaller than that between states in the same band. As can be seen from Eqs. (19) and (20) of Ref. 7, $W_{\zeta \zeta^{\prime}}$ is proportional to a factor $e^{-\gamma}$ which favors small values of $\gamma=l^{2}\left(q_{x}^{2}+q_{y}^{2}\right) / 2$. For states in the same band $\left(n=n^{\prime}\right)$ scattering broadening makes states with similar $k_{y}$ overlap in energy and $\gamma$ can be very small. Hopping between states with small $k_{y}$ differences contributes mainly to the collisional conductivity. However, for states in different bands $\left(n \neq n^{\prime}\right)$ which overlap in energy-i.e., $E_{n k_{y}}$ $=E_{n^{\prime} k_{y}^{\prime}}$ - the quantity $K x_{0}^{\prime}-K x_{0}=K l^{2}\left(k_{y}-k_{y}^{\prime}\right)$ cannot be very small, as can be seen from Eq. (3). For example, as adjacent bands $\left(n^{\prime}=n \pm 1\right)$ overlap, as shown in Fig. 3(a), states at the band edges have similar energies where $\mid K x_{0}^{\prime}$ $-K x_{0} \mid \approx(2 m+1) \pi, \quad m=0, \pm 1, \pm 2, \ldots ;$ thus, $\left|q_{y}\right|=\mid k_{y}$ $-k_{y}^{\prime}|=| x_{0}^{\prime}-x_{0} \mid / l^{2} \geqslant a / 2 l^{2} \quad$ and $\quad \gamma=l^{2}\left(q_{x}^{2}+q_{y}^{2}\right) / 2 \geqslant l^{2} q_{y}^{2} / 2$ $\geqslant(a / l)^{2} / 8$. For a sample with $a=500 \mathrm{~nm}$ and $B_{0} \simeq 0.1 \mathrm{~T}$ we obtain $\gamma \simeq 5$ and consequently the transition rate $W_{\zeta \zeta^{\prime}} \propto e^{-5}$ $\approx 0.007$ is very small. The physical reason is that the centers of the hopping states are far apart in space and the related wave function overlap is small. Omitting contributions from these interband hoppings in the collisional conductivity we get

$$
\begin{aligned}
\sigma_{x x}^{c o l}= & \frac{e^{2}}{h} \Gamma^{2} \int_{-\infty}^{\infty} d E\left(-\frac{\partial f}{\partial E}\right) \sum_{n=0}^{\infty}(2 n+1) \\
& \times \int_{0}^{\pi} d t P^{2}\left(E-E_{n t}\right) .
\end{aligned}
$$

The collisional conductivity in the $y$ direction equals that in the $x$ direction, $\sigma_{y y}^{c o l}=\sigma_{x x}^{c o l}$, in the first-order perturbation calculation as in Eq. (12). In the case that the thermal broadening is larger than the impurity broadening, $k_{B} T \gg \Gamma$, $(-\partial f / \partial E) \approx(-\partial f / \partial E)_{E=E_{n t}}$, and the integration over $E$ can be done analytically. We obtain

$$
\sigma_{x x}^{c o l}=\frac{e^{2}}{h} \frac{\Gamma}{2} \sum_{n=0}^{\infty}(2 n+1) \frac{1}{\pi} \int_{0}^{\pi} d t\left(-\frac{\partial f}{\partial E}\right)_{E=E_{n t}},
$$

which, apart from a factor of $1 / 2$, is Eq. (23) of Ref. 7. To investigate $\mathrm{SdH}$ oscillations in low temperatures $\left(k_{B} T\right.$ $\left.<W_{n}, \Gamma\right)$ we performed our numerical calculation using Eqs. (10) and (12).

As we showed above, transitions between states in different Landau bands have very small probabilities. Perturbation corrections to the wave function can be omitted because they involve electronic states from different Landau bands. The even-odd transitions in the resistivity, which are the main focus of the present work, will not be influenced by these corrections. They are related to the even-odd transitions in the DOS. The validity of the above perturbation theory was demonstrated from calculations with direct diagonalization of the Hamiltonian. ${ }^{3,6,15,28}$ Here we would like to point out that in the regime where Landau bands overlap, i.e., $W_{n} / \hbar \omega_{c}>1$, the calculated energy spectrum and resistance in Ref. 28 show rather good agreement with those obtained from the perturbation theory until a much larger modulation strength $V_{0} / \hbar \omega_{c} \approx 9.5$. The reason behind this is that the energy overlapping states in different Landau bands have different $k_{y}$. Hybridization of these states due to 1D modulations is forbidden. Clear deviation from a perturbation calculation occurs as the modulation is so strong that bound states (snake orbits) are formed near the local minima of the modulation potential. ${ }^{15,28,29}$

\section{COMPARISON WITH EXPERIMENTAL RESULTS}

In this section, calculated magnetoresistivities $\rho_{x x}$ and $\rho_{y y}$ are compared to the experimental results presented in Ref. 25. The samples considered are GaAs/AlGaAs Hall bars of width $50 \mu \mathrm{m}$, with voltage probes separated by $130 \mu \mathrm{m}$, and with a 2 DEG located $35 \mathrm{~nm}$ below the surface. The modulation results from the perpendicular component of the fringing magnetic fields of periodic arrays of ferromagnetic Co strips, which are fabricated on the surface of the Hall bars. A full description of the experiment is given elsewhere. ${ }^{25}$

For sample S1, the Co strips run perpendicular to the current direction, so that the magnetoresistance parallel to the modulation direction, $R_{x x}$, is measured. The strips are of height $120 \mathrm{~nm}$, width $200 \mathrm{~nm}$, and period $500 \mathrm{~nm}$, and the electron density $n_{e}=3.88 \times 10^{15} \mathrm{~m}^{-2}$. The measured $R_{x x}$ is shown in Fig. 5(a). The dotted curve is measured at a temperature $T=4.2 \mathrm{~K}$ and the solid curve at $80 \mathrm{mK}$. At $4.2 \mathrm{~K}$, only Weiss oscillations are observed, with minima occurring at $B_{0}=0.11,0.14,0.22$, and $0.42 \mathrm{~T}$, respectively. At $80 \mathrm{mK}$, both $\mathrm{SdH}$ and Weiss oscillations are present. In Fig. 5(b) the calculated magnetoresistivity $\rho_{x x}$ at $4.2 \mathrm{~K}$ (dotted curve) and at $80 \mathrm{mK}$ (solid curve) is shown. The amplitudes and phase of the Weiss and $\mathrm{SdH}$ oscillations at both temperatures are well reproduced. Except for a constant shift of $50 \Omega$ the calculated resistivities are in good agreement with the experimental ones. This shift may be a consequence of approximations made in our calculation, such as short-range scattering by impurities, zero-order wave functions in the transition rate, the omission of hopping between Landau bands, etc.

We assume that the perpendicular component of magnetization of the strips, and therefore the strength of the periodic 


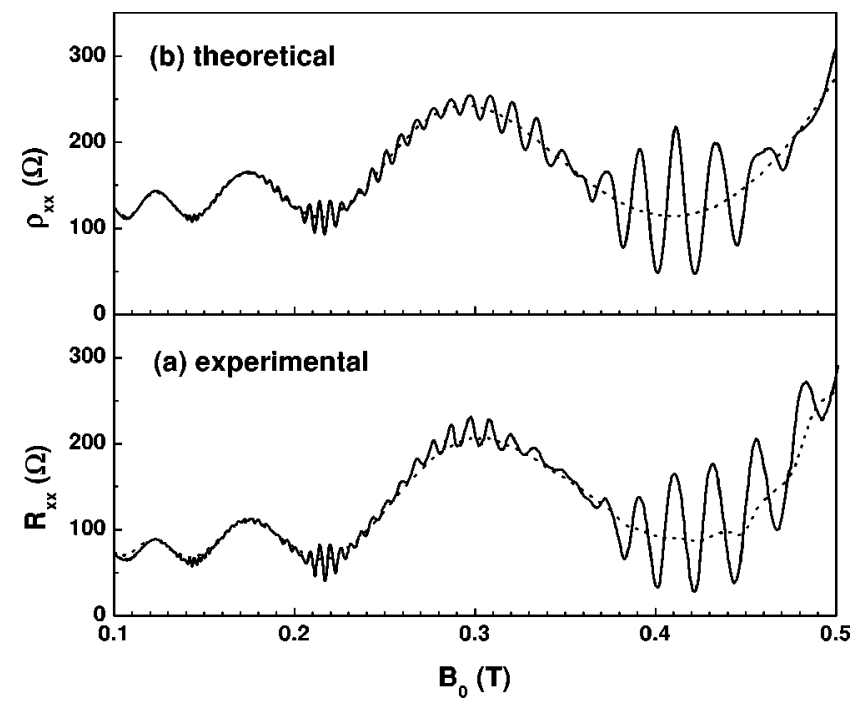

FIG. 5. (a) Experimental magnetoresistance and (b) theoretical magnetoresistivity along the modulation direction, $R_{x x}$, at $80 \mathrm{mK}$ (solid curves) and $4.2 \mathrm{~K}$ (dotted curves), respectively.

magnetic modulation $B_{1}$, increases linearly with the external field $B_{0}$ from zero up to a saturation value $B_{0}^{s}$, as expected for a Stoner-Wohlfarth single domain particle magnetized perpendicular to its axis. ${ }^{30}$ In the calculation we set $B_{1}$ $=0.1 B_{0}$ and $B_{0}^{s}=1.4 \mathrm{~T}$ in good agreement with the values $B_{1}=0.12 B_{0} \quad$ and $B_{0}^{s}=1.4 \mathrm{~T}$ estimated from the experiment. ${ }^{25}$ An in-phase electric modulation was included in order to incorporate the effect due to strain. This electric modulation affects the phase of the Weiss oscillations, which allows an independent determination of $V_{0}$ and $B_{1}{ }^{14}$ Without a suitable $V_{0}$, the measured positions of the Weiss oscillation minima cannot be reproduced. We set $V_{0}=0.8 \mathrm{meV}$ which agrees with that estimated from the measured lowfield positive magnetoresistance. ${ }^{25}$ Additional parameters used are the scattering broadening $\Gamma=2.7 \mathrm{~K}$ and the mo-

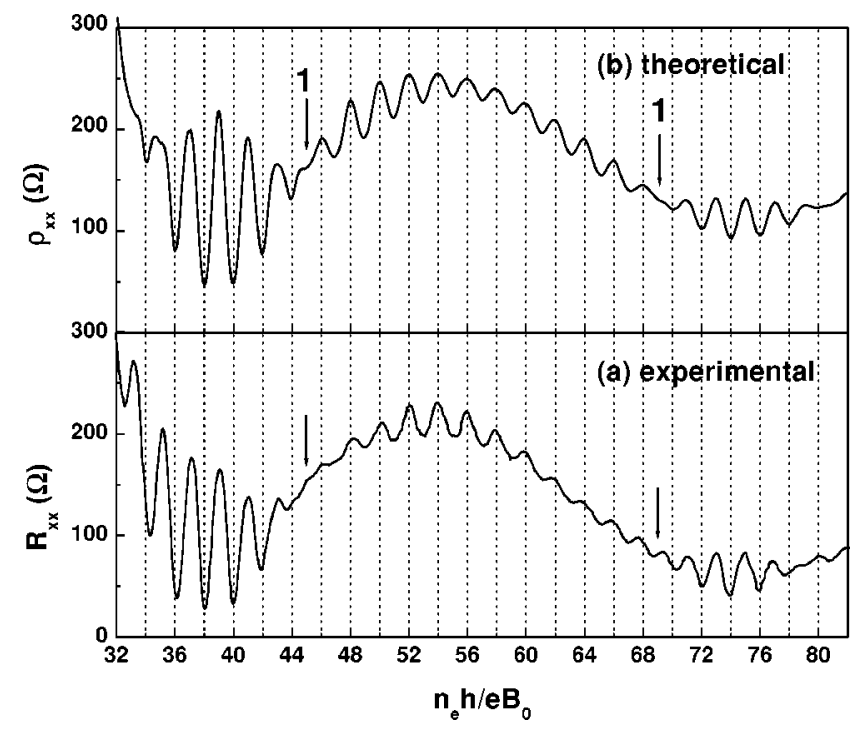

FIG. 6. (a) Experimental $R_{x x}$ and (b) theoretical $\rho_{x x}$ vs Landaulevel filling factor. Even-odd transitions are marked by the arrows.

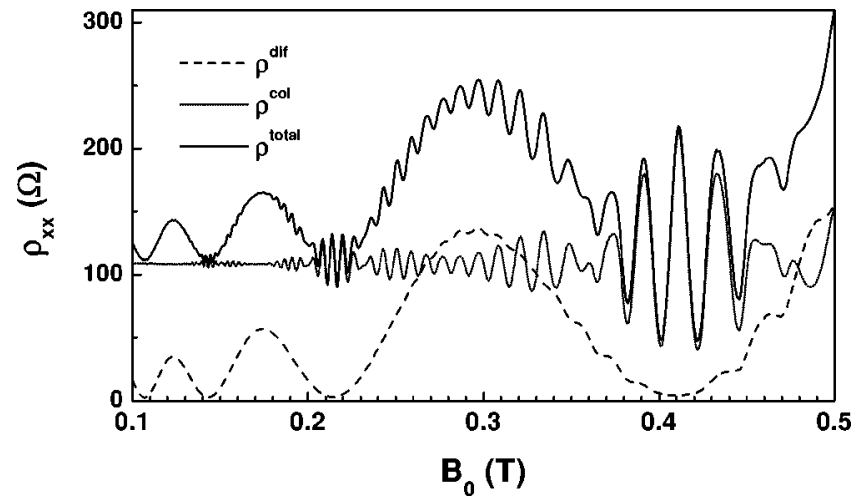

FIG. 7. Calculated diffusion (dashed curve) and collisional (dotted curve) contributions to the magnetoresistivity $\rho_{x x}$ (solid curve).

mentum relaxation time $\tau=3.66 \times 10^{-11} \mathrm{~s}$. The amplitudes of the $\mathrm{SdH}$ and Weiss oscillations are closely related to the value of $\Gamma$ and $\tau$. The momentum relaxation time evaluated from the mobility of the sample is $2.7 \times 10^{-11} \mathrm{~s}$.

The phases of the $\mathrm{SdH}$ oscillations are made more visible in Fig. 6 where we plot $\rho_{x x}$ as a function of the Landau level filling factor, $\nu=n_{e} h / e B_{0}$, in the magnetic field range $0.19 \mathrm{~T}<B_{0}<0.5 \mathrm{~T}$. The phase of the calculated SdH oscillation in Fig. 6(b) and that of the experimental result in Fig. 6(a) are in excellent agreement. Near the Weiss oscillation minima $\nu \approx 38$ and $\nu \approx 74$, SdH minima occur at even values of $\nu$; i.e., $R_{x x}$ reaches a minimum when the Fermi level lies between Landau levels, as is the case in a uniform magnetic field. Around the Weiss oscillation peak at $\nu \approx 54$, the $\mathrm{SdH}$ minima occur at odd numbers of $\nu$; i.e., $R_{x x}$ reaches a minimum when $E_{F}$ is at the center of a Landau band. The switching between the two oscillation modes, marked by the arrows in the figures, occurs at $\nu \approx 45$ and $\nu \approx 69$, on either side of the Weiss oscillation peak. This even-odd transition is related to the overlap of adjacent van Hove singularities, as dis-

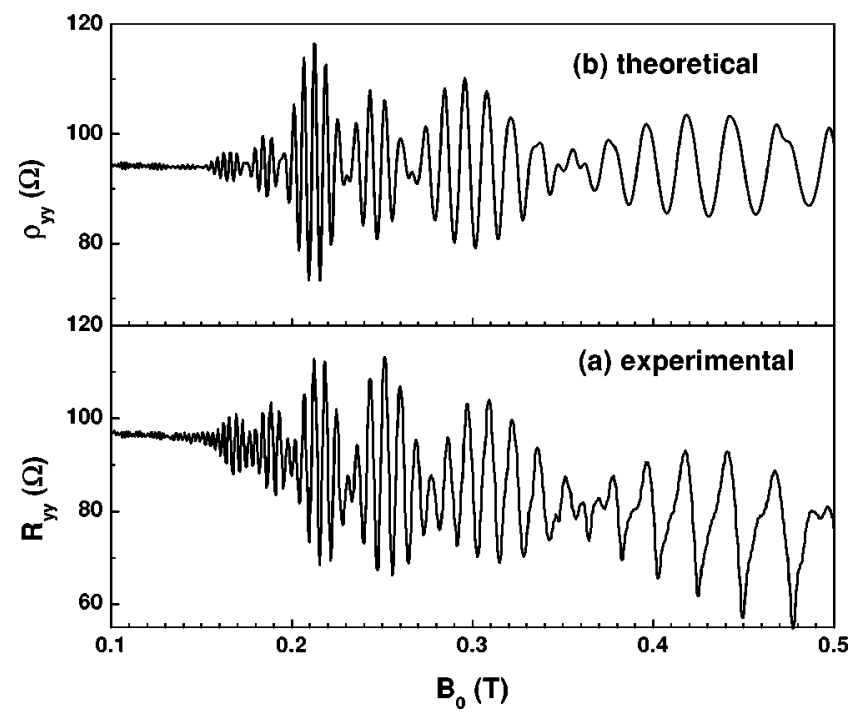

FIG. 8. (a) Experimental magnetoresistance $R_{y y}$ and (b) theoretical magnetoresistivity $\rho_{y y}$ perpendicular to the modulation direction at $0.3 \mathrm{~K}$. 


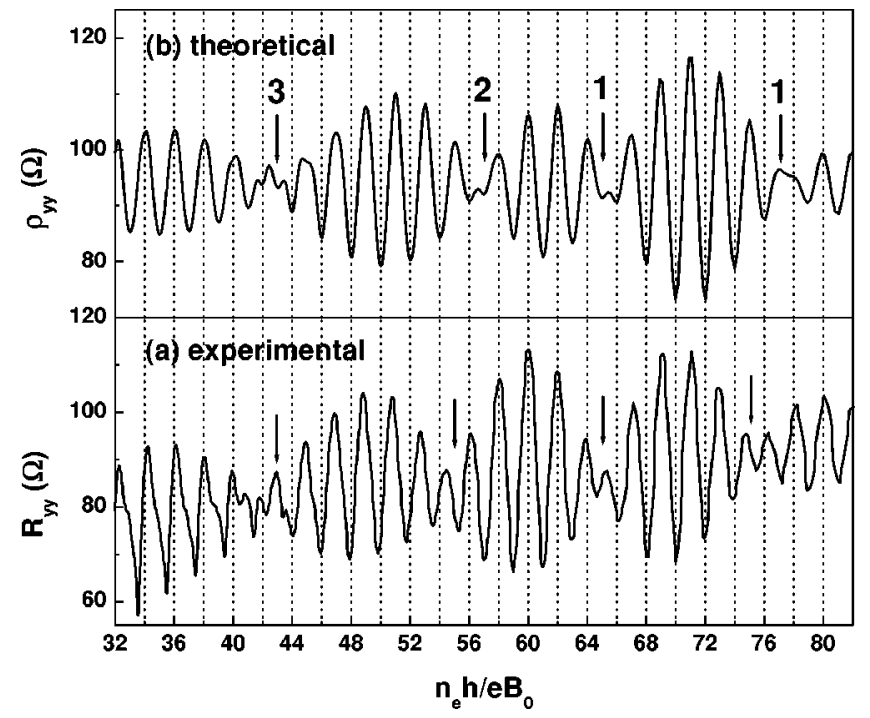

FIG. 9. (a) Experimental $R_{y y}$ and (b) theoretical $\rho_{y y}$ vs Landaulevel filling factor. Even-odd transitions are marked by the arrows. The numbers above the arrows in (b) are the Landau-band width at the Fermi energy in unit of $\hbar \omega_{0}$.

cussed in Sec. II. The calculated Landau-band width is equal to $\hbar \omega_{0}$ at the transition points.

In Fig. 7 we show the calculated diffusive resistivity $\rho_{x x}^{d i f}$ (dashed curve), the collisional resistivity $\rho_{x x}^{c o l}$ (dotted curve), and the total $\rho_{x x}$ (solid curve) at $T=80 \mathrm{mK}$. It is apparent that $\rho_{x x}^{\text {dif }}$ gives the dominant contribution to the Weiss oscillations while $\rho_{x x}^{c o l}$ gives the dominant contribution to the $\mathrm{SdH}$ oscillations. At low temperatures, the energy broadening due to scattering, $\Gamma$, smears the $\mathrm{SdH}$ oscillations in $\rho_{x x}^{d i f}$, while the $\mathrm{SdH}$ oscillations in $\rho_{x x}^{c o l}$ can survive to larger $\Gamma$. The Weiss oscillation in $\rho_{x x}^{c o l}$ is much weaker than in $\rho_{x x}^{d i f}$. The physical reason is that the former comes from the modulation effect on the DOS while the latter results from the diffusive motion of electrons in modulated fields. The collisional contribution is responsible for the stronger $\mathrm{SdH}$ oscillations near the minima of the Weiss oscillations.

In sample S2, the strips are aligned parallel to the current direction, so that the resistance perpendicular to the modulation direction, $R_{y y}$, is measured. In this case the strips are of height $60 \mathrm{~nm}$, width $400 \mathrm{~nm}$, and period $1000 \mathrm{~nm}$, with an electron density $n_{e}=3.65 \times 10^{15} \mathrm{~m}^{-2}$. The measured $R_{y y}$ at $0.3 \mathrm{~K}$ is shown in Fig. 8(a). The calculated $\rho_{y y}$ is shown in Fig. 8(b). In the calculation we set $V_{0}=0.8 \mathrm{meV}$ and $\Gamma$ $=2.2 \mathrm{~K}$. The magnetic modulation strength $B_{1}=0.065 B_{0}$ agrees well with the value obtained from experiments with external magnetic field at different angles to the sample, $B_{1}$ $=0.064 B_{0} .{ }^{25}$ The observed features in $R_{y y}$ are reasonably well reproduced by our calculation. The $\mathrm{SdH}$ oscillations dominate both in Figs. 8(a) and 8(b). The Weiss oscillations are very weak and hardly visible, since the diffusive contribution to $\rho_{y y}$ is equal to zero. In low fields $B_{0}<0.15 \mathrm{~T}$, the amplitude of the $\mathrm{SdH}$ oscillation is small. Above $0.15 \mathrm{~T}$, modulated $\mathrm{SdH}$ oscillations with nodes are observed. At $B_{0}$ $=0.17 \mathrm{~T}, 0.20 \mathrm{~T}, 0.23 \mathrm{~T}, 0.27 \mathrm{~T}$, and $0.35 \mathrm{~T}$ the SdH oscillations are strongly suppressed. The phase of the SdH oscil- lations changes at these nodes. This is seen more clearly in Fig. 9 where $R_{y y}$ is plotted as a function of the Landau filling factor $\nu=h n_{e} / e B_{0}$, over the magnetic field range $0.18 \mathrm{~T}$ $<B_{0}<0.47 \mathrm{~T}$. The experimental and calculated results are shown in Figs. 9(a) and 9(b), respectively. The positions of the $\mathrm{SdH}$ oscillation minima and maxima switch between even and odd values of $\nu$ in both figures. The even-odd transition points (the nodes) are indicated by the arrows in Fig. 9. In the calculated $\rho_{y y}$, these occur where the Landau-band width at the Fermi energy, $W$, is equal to an integer multiple of $\hbar \omega_{0}$; i.e., phase switching of the SdH oscillations occurs whenever the number of overlapping Landau bands at the Fermi energy changes. The number of overlapping bands at the transition points is marked above the arrows in Fig. 9(a). There are only small discrepancies between the values of $\nu$ in the theory and the experiment.

In Ref. 25 very weak Weiss oscillations and even-odd transitions in the $\mathrm{SdH}$ oscillations were also found in the Hall resistance $\left(R_{H}\right)$. After subtracting the linear term in $R_{H}$ the obtained oscillations were an order of magnetude smaller than those in $R_{x x}$. Such oscillations can also be induced by a small misalignment of the Hall probes and this issue has to be cleared out before any detailed theoretical comparison is meaningful.

\section{CONCLUSIONS}

We have investigated magnetoresistance oscillations of a 2DEG in the presence of one-dimensional periodic magnetic and electric fields. The previous quantum perturbation theory ${ }^{14}$ was generalized to low temperatures and large magnetic and electric modulation amplitudes. In addition, the energy broadening effect due to impurity scattering is considered which is important at low temperatures $(T<1 \mathrm{~K})$. With reasonable parameter values our calculation results agree well with recent experiments. The $\mathrm{SdH}$ oscillations in the resistances $R_{x x}$ and $R_{y y}$ are mostly due to the $\mathrm{SdH}$ oscillations in the collisional contribution to the conductivity. The $\mathrm{SdH}$ oscillations were found to be more pronounced near the minima of the Weiss oscillations. The $\mathrm{SdH}$ oscillations in $R_{x x}$ and $R_{y y}$ exhibit phase shifts which lead to minima (and maxima) at even or odd filling factor. This even-odd transition is a consequence of overlapping Landau levels which are broadened by the strong magnetic and electric modulation. The even-odd transition occurs when the width of the Landau band is an integer multiple of the cyclotron energy.

\section{ACKNOWLEDGMENTS}

This work was supported by the Flemish Science Foundation (FWO-Vl), IUAP-IV, the "Onderzoeksraad van de Universiteit Antwerpen" (GOA), the Bilateral Scientific and Technological Cooperation between Flanders and China, and the EPSRC (UK).

\section{APPENDIX}

We present in this appendix the calculation of the fringe magnetic field of a periodic array of ferromagnetic strips and 
make a Fourier analysis of the magnetic field profiles. Since no conduction current is involved, Maxwell's equations can be written as

$$
\vec{\nabla} \cdot \vec{B}=0, \quad \vec{\nabla} \times \vec{H}=0,
$$

where $\vec{B}=\mu_{0}(\vec{H}+\vec{M})$ and $\vec{M}$ is the magnetization. In this case a magnetic scalar potential $\Phi_{m}$ can be introduced with $\vec{H}=-\vec{\nabla} \Phi_{m}$. If a magnetic charge density is defined as $\rho_{m}$ $=-\vec{\nabla} \cdot \vec{M}$, we obtain Poisson's equation

$$
\nabla^{2} \Phi_{m}=-\vec{\nabla} \cdot \vec{H}=\vec{\nabla} \cdot \vec{M}=-\rho_{m},
$$

which can be solved by standard methods in electrostatics. ${ }^{31}$

We start the calculation with the scalar potential of a magnetic dipole

$$
\Phi_{d}(\vec{r})=\frac{1}{4 \pi} \frac{\vec{p} \cdot \vec{r}}{|\vec{r}|^{3}},
$$

where $\vec{p}$ is the magnetic moment of the dipole. The scalar potential of a uniformly magnetized strip along the $y$ axis is calculated as

$$
\Phi_{m}(\vec{r})=\int_{-h / 2}^{h / 2} d z^{\prime} \int_{-d / 2}^{d / 2} d x^{\prime} \int_{-\infty}^{\infty} d y^{\prime} \Phi_{d}\left(\vec{r}-\vec{r}^{\prime}\right),
$$

where $d$ and $h$ are the thickness and height of the strip, respectively. For the case when magnetization is in the $z$ direction, i.e., $\vec{M}=M \vec{e}_{z}$, we obtain

$$
\begin{aligned}
\Phi_{m}(x, z)= & f\left(x-\frac{d}{2}, z-\frac{h}{2}\right)-f\left(x-\frac{d}{2}, z+\frac{h}{2}\right) \\
& -f\left(x+\frac{d}{2}, z-\frac{h}{2}\right)+f\left(x+\frac{d}{2}, z+\frac{h}{2}\right),
\end{aligned}
$$

where

$$
f(x, z)=\frac{M}{2 \pi}\left[z \tan ^{-1}\left(\frac{x}{z}\right)+\frac{x}{2} \ln \left(x^{2}+z^{2}\right)\right] .
$$

The fringe magnetic field is evaluated as $\vec{B}=\mu_{0} \vec{H}=$ $-\mu_{0} \vec{\nabla} \Phi_{m}$. The $z$ component of the magnetic field, which affects the motion of the electrons in the $(x, y)$ plane, reads

$$
\begin{aligned}
B_{z}^{s}(x, z)= & -\frac{\mu_{0} M}{2 \pi}\left[\tan ^{-1}\left(\frac{x-d / 2}{z-h / 2}\right)-\tan ^{-1}\left(\frac{x-d / 2}{z+h / 2}\right)\right. \\
& \left.-\tan ^{-1}\left(\frac{x+d / 2}{z-h / 2}\right)+\tan ^{-1}\left(\frac{x+d / 2}{z+h / 2}\right)\right]
\end{aligned}
$$

The magnetic field resulting from a periodic array of strips with period $a$ is a superposition of contributions from individual strips:

$$
B_{z}(x, z)=\sum_{n=-\infty}^{\infty} B_{z}^{s}(x-n a, z)
$$

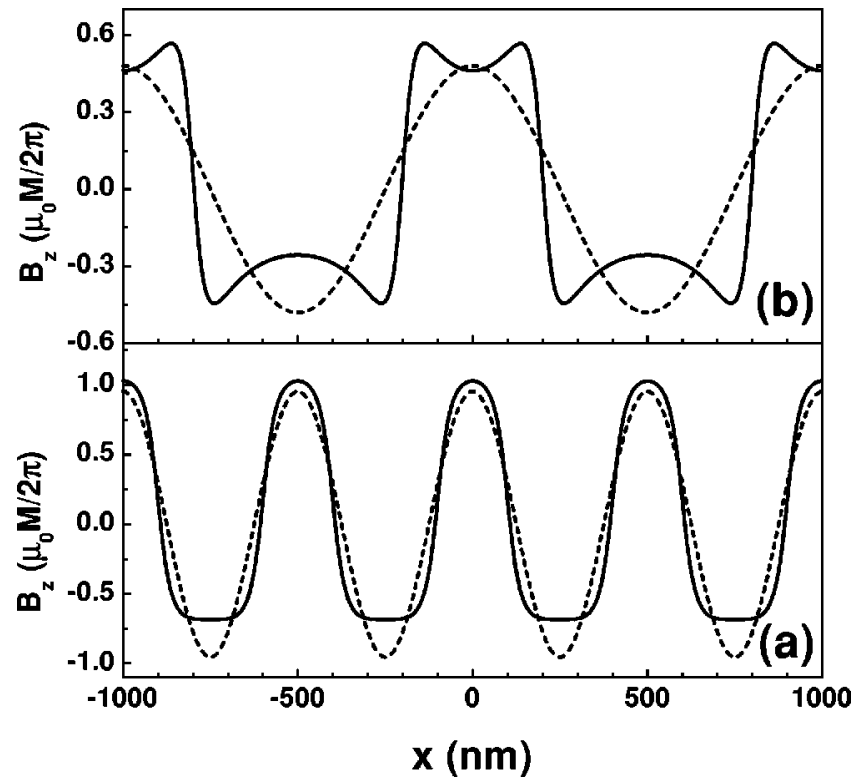

FIG. 10. Calculated fringe magnetic fields $B_{z}$ in the 2DEG resulting from periodic ferromagnetic strips along the $y$ direction (solid curves) and their first Fourier component (dashed curves). Panel (a) is for sample S1 in which the period of the strips (in the $x$ direction) is $a=500 \mathrm{~nm}$, the thickness $d=200 \mathrm{~nm}$, and the height (in the $z$ direction) $h=120 \mathrm{~nm}$. Panel (b) is for sample S2 where $a=1000 \mathrm{~nm}, d=400 \mathrm{~nm}$, and $h=60 \mathrm{~nm}$. The 2DEG is located $35 \mathrm{~nm}$ below the strips in both samples.

The $B_{z}$ obtained above is an even and periodic function of $x$. Thus we can make a Fourier expansion of the magnetic profile with cosine functions,

$$
B_{z}(x, z)=\frac{B^{(0)}}{2}+\sum_{n=1}^{\infty} B^{(n)}(z) \cos (n K x),
$$

where $K=2 \pi / a$ and

$$
B^{(n)}(z)=\frac{2}{a} \int_{-a / 2}^{a / 2} d x B_{z}(x, z) \cos (n K x), n=0,1,2, \ldots
$$

The average of a fringe magnetic field is always zero, i.e.,

$$
B^{(0)}=\frac{2}{a} \int_{-a / 2}^{a / 2} d x B_{z}(x, z)=\frac{2}{a} \int_{-\infty}^{\infty} d x B_{z}^{s}(x, z)=0 .
$$

In the absence of an external magnetic field, the magnetization of the strips of S1 and S2 will lie along their length due to magnetic shape anisotropy. Since the strips are much longer than the Hall bar and symmetrically placed, there will be no perpendicular component of the fringing magnetic fields of the strips in this case. Applying a magnetic field perpendicular to the Hall bar will produce a perpendicular component of the magnetization, $M_{z}$, and therefore a finite $B_{z}$.

The calculated $B_{z}$ for sample $\mathrm{S} 1$ and $\mathrm{S} 2$ in the experiments are shown by the solid curves in Fig. 10(a) and 10(b), 
respectively. Note that the magnetic field profiles are asymmetric about $B_{z}=0$. The Fourier analyses for these field profiles were made and their first Fourier components are also plotted in Fig. 10 by the dashed curves. The amplitudes of the first four components of $\mathrm{S} 1$ are $B^{(1)}=0.954, B^{(2)}$ $=0.232, B^{(3)}=-0.104$, and $B^{(4)}=-0.082$. A good approximation to $B_{z}$ can be made with the first two components $B^{(1)}$ and $B^{(2)}$. We calculated the DOS in this case $\left(B^{(2)} \neq 0\right)$ and compared it with that with only the first component $\left(B^{(2)}\right.$ $=0)$. There are only minor differences and they gave evenodd transitions at the same magnetic fields. As was shown in
Ref. 16, the contributions of the $n$th component to the DOS and to the conductivities are exponentially diminished by a factor of $e^{-n^{2} u}$ where $u=K^{2} l^{2} / 2$ and $l$ is the magnetic length. This is the reason why we are allowed to restrict ourselves to the $n=1$ component in our analysis. The amplitudes of the first four components of S2 are $B^{(1)}=0.480$, $B^{(2)}=0.201, B^{(3)}=-0.137$, and $B^{(4)}=-0.154$. In this case the first component is a poor approximation to the real magnetic profile. This may be one of the reasons that makes our calculated $\rho_{y y}$ for $\mathrm{S} 2$ deviate slightly with the experimental result.
*Electronic address: peeters@uia.ua.ac.be

${ }^{1}$ For a recent review, see F.M. Peeters and Jo De Boeck, in Handbook of Nanostructured Materials and Nanotechnology, edited by H.S. Nalwa (Academic Press, New York, 2000), Vol. 3, p. 345 .

${ }^{2}$ D. Weiss, K.v. Klitzing, K. Ploog, and G. Weimann, Europhys. Lett. 8, 179 (1989).

${ }^{3}$ R.R. Gerhardts, D. Weiss, and K.v. Klitzing, Phys. Rev. Lett. 62, 1173 (1989).

${ }^{4}$ R.W. Winkler, J.P. Kotthaus, and K. Ploog, Phys. Rev. Lett. 62, 1177 (1989).

${ }^{5}$ P. Vasilopoulos and F.M. Peeters, Phys. Rev. Lett. 63, 2110 (1989).

${ }^{6}$ C. Zhang and R.R. Gerhardts, Phys. Rev. B 41, 12850 (1990).

${ }^{7}$ F.M. Peeters and P. Vasilopoulos, Phys. Rev. B 46, 4667 (1992).

${ }^{8}$ C.W.J. Beenakker, Phys. Rev. Lett. 62, 2020 (1989).

${ }^{9}$ R.R. Gerhardts, Phys. Rev. B 45, 3449 (1992).

${ }^{10}$ R.R. Gerhardts, Phys. Rev. B 53, 11064 (1996).

${ }^{11}$ R. Menne and R.R. Gerhardts, Phys. Rev. B 57, 1707 (1998).

${ }^{12}$ P. Vasilopoulos and F.M. Peeters, Superlattices Microstruct. 7, 393 (1990).

${ }^{13}$ D.P. Xue and G. Xiao, Phys. Rev. B 45, 5986 (1992).

${ }^{14}$ F.M. Peeters and P. Vasilopoulos, Phys. Rev. B 47, 1466 (1993).

${ }^{15}$ R. Yagi and Y. Iye, J. Phys. Soc. Jpn. 62, 1279 (1993).

${ }^{16}$ T. Li, S. Gu, X. Wang, and J. Peng, J. Phys.: Condens. Matter 8, 313 (1996).

${ }^{17}$ H.A. Carmona, A.K. Geim, A. Nogaret, P.C. Main, T.J. Foster, M. Henini, S.P. Beaumont, and M.G. Blamire, Phys. Rev. Lett. 74, 3009 (1995).
${ }^{18}$ P.D. Ye, D. Weiss, R.R. Gerhardts, M. Seeger, K. von Klitzing, K. Eberl, and H. Nickel, Phys. Rev. Lett. 74, 3013 (1995).

${ }^{19}$ Y. Iye, A. Endo, S. Izawa, M. Kato, and S. Katsumoto, Physica B 227, 122 (1996).

${ }^{20}$ A. Nogaret, S. Carlton, B.L. Gallagher, P.C. Main, M. Henini, R. Wirtz, R. Newbury, M.A. Howson, and S.P. Beaumont, Phys. Rev. B 55, R16 037 (1997).

${ }^{21}$ N. Overend, A. Nogaret, B.L. Gallagher, P.C. Main, M. Henini, C.H. Marrows, M.A. Howson, and S.P. Beaumont, Appl. Phys. Lett. 72, 1724 (1998).

${ }^{22}$ J.H. Davies and I.A. Larkin, Phys. Rev. B 49, 4800 (1994).

${ }^{23}$ I.A. Larkin, J.H. Davies, A.R. Long, and R. Cuscó, Phys. Rev. B 56, 15242 (1997).

${ }^{24}$ A.R. Long, E. Skuras, S. Vallis, R. Cuscó, I.A. Larkin, J.H. Davies, and M.C. Holland, Phys. Rev. B 60, 1964 (1999).

${ }^{25}$ K.W. Edmonds, B.L. Gallagher, P.C. Main, N. Overend, R. Wirtz, A. Nogaret, M. Henini, C.H. Marrows, B.J. Hickey, and S. Thoms, Phys. Rev. B 64, 041303 (2001).

${ }^{26}$ M. Tornow, D. Weiss, A. Manolescu, R. Menne, and K. von K1itzing, Phys. Rev. B 54, 16397 (1996).

${ }^{27}$ M. Charbonneau, K.M. Van Vliet, and P. Vasilopoulos, J. Math. Phys. 23, 318 (1982).

${ }^{28}$ Q.W. Shi and K.Y. Szeto, Phys. Rev. B 53, 12990 (1996).

${ }^{29}$ J. Reijniers and F.M. Peeters, J. Phys.: Condens. Matter 12, 9771 (2000).

${ }^{30}$ V. Kubrak, F. Rahman, B.L. Gallagher, P.C. Main, M. Henini, C.H. Marrows, and M.A. Howson, Appl. Phys. Lett. 74, 2507 (1999).

${ }^{31}$ I.S. Ibrahim and F.M. Peeters, Phys. Rev. B 52, 17321 (1995). 